\title{
Evidence of an advantage in visuo-spatial memory for bilingual compared to monolingual speakers
}

\begin{tabular}{|r|l|}
\hline Journal: & Bilingualism: Language and Cognition \\
\hline Manuscript ID & BLC-15-RA--0080.R2 \\
\hline Manuscript Type: & Research Article \\
\hline Complete List of Authors: & Kerrigan, Lucy; Anglia Ruskin University, Psychology \\
& $\begin{array}{l}\text { Thomas, Michael; Birkbeck, Psychological Sciences } \\
\text { Bright, Peter; Anglia Ruskin University, Psychology } \\
\text { Filippi, Roberto; Anglia Ruskin University, Psychology }\end{array}$ \\
\hline Content Areas: & Cognitive Psychology \\
\hline Linguistic Areas: & Not Applicable \\
\hline Methods: & Behavioural Measurements \\
\hline Populations: & Healthy Normal Subjects \\
\hline \hline
\end{tabular}


2

\section{Highlights}

- We compared bilingual and monolingual speakers on a range of working memory tasks

- We found a bilingual advantage in processing visuo-spatial information

- Unlike previous findings, there was no bilingual disadvantage in verbal processing

- Bilingualism may enhance attentional control processing for non-verbal information 
Running head: Bilingualism and Working Memory

\title{
Evidence of an advantage in visuo-spatial memory for bilingual compared to monolingual speakers
}

\author{
Lucy Kerrigan $^{1}$, Peter Bright ${ }^{1}$, Michael, S. C. Thomas ${ }^{2}$, Roberto Filippi ${ }^{1,2}$ \\ ${ }^{1}$ Anglia Ruskin University, Cambridge \\ 2 Developmental Neurocognition Lab, Birkbeck, University of London
}

Address for correspondence

Dr Roberto Filippi

Anglia Ruskin University

Department of Psychology

Faculty of Science \& Technology

East Road

Cambridge CB1 1PT

Email: roberto.filippi@anglia.ac.uk

Word count: 5,555 (references excluded)

Acknowledgements: This study was funded by the Leverhulme Trust, grant RPG2015-024 and UK ESRC grant RES-062-23-2721. Our thanks to Prof. Fergus Craik for his valuable input. 


\begin{abstract}
Previous research has indicated that bilinguals outperform monolinguals in cognitive tasks involving spatial working memory. The present study examines evidence for this claim using a different and arguably more ecologically valid method (the change blindness task). Bilingual and monolingual participants were presented with two versions of the same scenes and required to press a key as soon as they identified the alteration. They also completed the word and alpha span tasks, and the Corsi blocks task. The results in the change blindness task, controlled for group differences in nonverbal reasoning, indicated that bilinguals were faster and more accurate than monolinguals at detecting visual changes. Similar group differences were found on the Corsi block task. Unlike previous findings, no group differences were found on the verbal memory tasks. The results are discussed with reference to mechanisms of cognitive control as a locus of transfer between bilingualism and spatial working memory tasks.
\end{abstract}

Keywords: Bilingualism, Executive Function, Working Memory, Verbal memory, Visuo-spatial memory, Visual detection. 


\section{Introduction}

In a progressively mobile world, the need for communicating in more than one language has become increasingly important. Over half of the world's population more than 3 billion people - is estimated to be bilingual (Grosjean, 2010) and twothirds of children in the world are growing up in multilingual environments (Crystal, 1997). Historically, second language learning was seen as detrimental for cognitive development. Early studies indicated that those acquiring a second language typically had lower scores in IQ tests than monolingual counterparts (e.g., Saer, 1923), thereby encouraging the common belief that bilingualism is " $b a d$ ". Educators tended to discourage second language learning early in life, arguing that the high cognitive demand of learning two sets of vocabulary and grammar would in turn cause a general developmental delay (Hakuta \& Diaz, 1985)

However, recent evidence offers a different perspective on cognitive change associated with bilingualism (for a review, see Bialystok, 2009). A much closer and systematic investigation on second language acquisition has now become part of main-stream psychology. Bilingualism is now offering a unique opportunity to study how language is acquired and how linguistic processes are intimately connected to general cognitive domains, such as memory and attention. In particular, research has focussed on executive function, namely, the ability to inhibit irrelevant information, shift between tasks and update the content from working memory (Miyake, Friedman, Emerson et al., 2000)

There is now robust evidence showing that bilingual speakers may have a cognitive advantage over monolinguals on executive function tasks requiring shifting targets and inhibiting irrelevant information (e.g., Bialystok, Craik, Green \& Gollan, 
2009). The advantage is observed with a variety of visual tasks (Bialystok, 1992;

Bialystok, Craik, Klein \& Viswanathan, 2004), auditory tasks (Filippi, Leech, Thomas, Green \& Dick, 2012; Vega-Mendoza, West, Sorace \& Bak, 2015), in children (Bialystok, 2001; Bialystok \& Martin, 2004; Carlson \& Meltzoff, 2008;

Filippi, Morris, Richardson et al., 2014), and adults who learned a second language late in life (Filippi, Richardson, Leech et al., 2011; Filippi et al., 2012). Evidence also indicates that this advantage is present throughout development until old age (Bialystok et al., 2004).

The current interpretation for this advantage is that in order to process one language, bilinguals need to suppress the other. This constant inhibitory mental "work-out" could in turn strengthen general executive function processes helping bilingual speakers block distracting information (Bialystok, 2009). This hypothesis would also explain evidence showing that a life-long use of two (or more) languages might offer some level of protection against cognitive deterioration associated with normal ageing as well as Alzheimer's disease and other age-related neurodegenerative conditions (e.g., Bak, Nissan, Allerhand and Deary, 2014; Craik, Bialystok \& Friedman, 2010). However, a more extensive body of research would be required to confirm this beneficial effect of bilingualism across different cultures.

There are some grounds to be sceptical of the reported benefits of bilingualism, since in research studies allocation of individuals to 'bilingual' versus 'monolingual' groups is not random. Therefore, bilingualism per se could index any number of sampling confounds, from intelligence to personality to the sorts of environmental stimulation that individuals receive across development. Moreover, claims of a bilingual advantage have been challenged by researchers who have found no evidence of differences in executive function across bilingual and monolingual 
groups (Duñabeitia, Hernández, Antón et al., 2013; Morton \& Harper, 2007; Paap \& Greenberg, 2013). Some authors have argued that studies reporting statistically significant results are more likely to be published causing a bias towards the so called "bilingual advantage" (de Bruin, Treccani \& Della Sala, 2014). Others argue that research favouring a bilingual cognitive advantage are based on single experimental tasks of questionable ecological validity. When a combination of multiple executive function tasks are employed within a single study, differences between monolinguals and bilinguals are not observed (Duñabeitia et al., 2013; Paap \& Greenberg, 2013). Therefore, the "bilingual advantage" might be caused by a task-specific artefact (Paap, Johnson \& Sawi, 2014). Perhaps the strongest grounds for scepticism that bilingualism is the active agent in observed bilingual/monolingual group differences is the claimed distance of transfer from bilingualism, a linguistic phenomenon, to other cognitive tasks outside the domain of language. Research on cognitive training typically finds that transfer effects are relatively near (e.g., Shipstead, Redick \& Engle, 2012; Wass, Scerif \& Johnson, 2012). Why should bilingualism be so much more powerful?

The unresolved debate on whether (and the extent to which) bilingualism offers a genuine cognitive advantage demands further targeted research in order to progress the development of theory. Currently perhaps the most influential bilingual framework linking general cognitive processes to language comprehension and production is the Inhibitory Control Model (ICM - Green 1986, 1998). This model assumes that during the phase of speech planning, a general mechanism controls speakers' communicative intentions. This mechanism derives from Norman and Shallice's (1986) model of action control. Green borrowed the term schema, a mental device that individuals employ or adapt in the service of specific behavioural or 
cognitive goals. Selection of a non-routine schema requires voluntary controlled action modulated by a "Supervisory Attentional System - SAS" (Norman \& Shallice, 1980). This system, equivalent to Baddeley's “central executive”, is part of the working memory model (Baddeley, 1986, 1992), responsible for the production of goal-directed behaviour. To the extent that the cognitive operations required of a bilingual enhance general control mechanisms that are utilized in other tasks, the ICM provides a mechanistic basis for the far transfer effects that have been associated with the bilingual advantage.

In this article, we consider possible transfer effects of bilingualism to shortterm and working memory. Previous research has not indicated reliable evidence for a difference in free recall performance between bilinguals and monolinguals. For example, Bialystok and Feng (2011) assessed simple verbal recall through a combined analysis of three experiments involving 1906 to 9 year old children, half of whom were bilingual speakers. The results show that there was no difference in children's ability to recall a list of animal names, in which word length became increasingly longer. However, the task did not require the need to manipulate the information being held in memory, such as in backwards serial recall. Hence, the authors described the data as a measure of short-term memory and concluded that retrieval from short-term memory is equivalent in bilingual and monolingual children. Additionally, this paradigm targeted a restricted aspect of memory specifically related to verbal information. In order to acquire a more comprehensive account of performance it may be instructive to employ additional tasks placing greater demand on working memory (including visuo-spatial abilities). Luo et al., (2013) measured both verbal and spatial performance in older and younger bilingual and monolingual adult speakers. The aim was not only to measure possible difference in working 
memory processes between monolinguals and bilinguals, but also to investigate whether these processes change across the lifespan, with bilingualism potentially offering some level of protection from the deleterious effects of age on cognition in older people.

To assess spatial working memory, Luo et al. used the Corsi blocks task (Milner, 1971), developed as a visuo-spatial counterpart to the verbal-memory span task. In the computerised version of this task, participants are presented with squares of the same colour (e.g., all blue) on the screen. At the experimenter's command, the squares changed to another colour (e.g., yellow) in sequence. The participants are then required to hold the sequence in mind and replicate it either forward (simple condition) or backward (complex condition) with the computer mouse.

To assess verbal working memory, Luo et al. used the word and alpha span tasks (Craik, 1986). In these tasks, the experimenter reads a list of common concrete nouns aloud which vary in length from two to eight words, with two lists of each length. In the word span task (simple condition), the participants have to recall the words in the same order. However, in the alpha span task (complex condition) they are recalled in alphabetical order.

The results surprisingly revealed that bilinguals outperformed monolinguals in the spatial working memory tasks, both for the simple and complex conditions. However, bilingual speakers achieved lower levels of performance than monolinguals on verbal working memory, reflected by a smaller number of recalled items in both the word span and alpha span tasks. These results are consistent with the view that bilinguals show a mild deficit in verbal processing compared to monolinguals, particularly with respect to lexical production, as reported in previous studies (e.g., 
Gollan, Montoya, Fennema-Notestine \& Morris, 2005); but suggested that they might have an advantage in spatial tasks, where no retrieval of verbal materials is required (Bialystok et al., 2008; 2009). Luo and colleagues predicted that there would be a greater effect of age on spatial working memory tasks than verbal working memory tasks. However, this was not borne out by the data, which instead indicated that ageing was associated with poorer working memory performance in both domains, and in both monolingual and bilingual groups. At face value, these findings counter claims that bilingualism offers protection against the effects of ageing on cognition (e.g., Craik et al., 2010). Nevertheless, they provide further evidence of the surprising far transfer effects of bilingualism outside the language domain.

In the present study, we sought further evidence that bilingualism confers an advantage on spatial working memory. The study built on Luo et al.'s (2013) investigation but incorporated an arguably more ecologically valid measure of visuospatial working memory, the change blindness task (Grimes 1996; Rensink 2002; Simons \& Rensink, 2005). Change blindness refers to the inability to detect important visual changes that occur during scene transitions. The term change-detection relates to the visual processes involved in first noticing a change, and being aware of the stimuli presented. It denotes not only identification (what the change is) but also localisation (where the change is; Rensink, 2002).

The performance of a group of young monolingual English adults was compared with age-matched bilinguals of different linguistic background on a battery of working memory tests. Our method enhanced ecological validity by systematically exposing participants to typical everyday visuo-spatial memory elements from which they were required to detect change. Visual detection changes in everyday scenes are 
not only important for accurate visual processing of the physical environment, but also promote general operational safety. Although research has defined changeblindness by the failure to store visual information in short-term memory (Rensink, O’Regan \& Clark, 1997; Rensink, 2002), limits in spatial memory capacity may also underpin the process of change detection (Rensink et al., 1997; Simons \& Levin, 2003). For an individual to successfully identify a change, focused attention and encoding is required (Hollingworth \& Henderson, 2002; Levin \& Simons, 1997). Alongside this, visuo-spatial working memory is needed in order to successfully compare first and second presentation of stimuli (Mitroff, Simons \& Levin, 2004; Simons \& Rensink, 2005).

During this task an original and modified image are presented in rapid alternation. The participants' aim is to respond as soon as they detect the modified element. Rensink et al. (1997) found three particular patterns of behaviour. First, it is rare that participants detect changes during the first alternation of images. Secondly, changes are not often detected after one minute of alternations and, thirdly, changes in the objects in the 'centre of interest' are identified more quickly than changes in the peripheral line of vision.

The present study sought further evidence of whether bilingualism is associated with an enhancement in the ability to process visuo-spatial and/or verbal information. Given the bilingual advantage observed in Luo's and colleagues' study, it was predicted that bilingual participants would outperform monolingual speakers in visuo-spatial processing and that this advantage would be found also with a more ecological task that mimics visual input from everyday life. However, on the basis of 
the existing literature, we did not expect a bilingual advantage in verbal working memory processing.

\section{Method}

\section{Participants}

Sixty undergraduate full time university students, 30 bilinguals (mean age $=21$ years old, $\mathrm{SD}=2.1$ ) and 30 English monolinguals (mean age $=22$ years old, $\mathrm{SD}=2.2$ ), were included in this study. All bilingual participants learnt English as a second language, but had different first language backgrounds [Greek $(n=15)$, Malay $(n=4)$, Mandarin $(n=2)$, Turkish $(n=2)$, Portuguese $(n=1)$, Japanese $(n=1)$, Igbo-African $(n=1)$, Bulgarian $(n=1)$, Spanish $(n=1)$, and Polish $(n=2)]$. All monolingual participants were native speakers of English only, had studied a second language at school, but no longer used it on a daily basis. All participants had normal or corrected-to-normal vision.

The bilingual participants completed a Language History Questionnaire (LHQ) (Filippi et al., 2012, 2013, 2014) in which they provided additional information on their second language acquisition experience. They were all living in the UK at the time of testing, and reported a balanced used of both their L2 and L1 on a daily basis (i.e., approximately equal amount of use of English and their native language). On average, they were first exposed to English from the age of eight. All bilingual participants self-rated their competence in English on four language dimensions using a scale ranging from 1 (very poor) to 6 (native-like). All reported very good competence in English on all dimensions, with a 5.0 mean score for reading ability $(\mathrm{SD}=0.9), 5.0$ for writing ability $(\mathrm{SD}=1.0), 5.2$ for listening ability $(\mathrm{SD}=0.8)$ and 5.2 for speaking ability $(\mathrm{SD}=0.8)$. From this, all participants were 
admitted to take part in the study. Out of the thirty bilingual participants, ten reported being exposed to a third or fourth language, but only four participants rated themselves as fluent in three languages and two fluent in four languages.

\section{Tasks and Procedure}

All participants were individually tested in a quiet room. The full battery of tasks took approximately 45 minutes to complete.

All participants completed two tests designed to assess visuo-spatial memory (the change blindness task (Rensink, et al., 1997) and the Corsi blocks task - forwards and backwards (Milner, 1971; Vandierendonck, Kemps, Fastame et al., 2004), two measures of verbal memory (the word and alpha span task (Craik, 1986) and a measure of non-verbal reasoning (the Cattell Culture Fair Test, Scale 2, Form A; Cattell, 1973).

\section{The change blindness task}

The design and procedure was adapted from Rensink et al. (1997). Pictures were presented using a Dell Inspiron laptop, with a 15.6-inch widescreen display and $800 \times 600$ resolution. Participants were seated approximately $70 \mathrm{~cm}$ from the screen. Data scores (response time and accuracy) were automatically recorded using E-Prime 2.0 (Schneider, Eschman \& Zuccolotto, 2002).

Participants were shown ten colour everyday life scenes. Each image was paired with a modified image and presented at a rate of $250 \mathrm{~ms}$ each. There was a gap of $1000 \mathrm{~ms}$ between repetitions of alternations, during which a black screen was presented. The modified image had one element missing. The missing element varied in size, colour and spatial location within the scene (Rensink et al., 1997). Figure 1 
illustrates an example of two images used in a trial. Once the participant had identified the visual change, they were asked to press the space bar as quickly as possible. This action paused the experiment and allowed for a ten-second rest. During this time, the participants were asked to verbally identify the change, and the experimenter noted whether they had correctly identified it or not. Participants were given 1 minute to identify the visual change. If they ran out of time, the experimenter pressed the space bar to move on to the next trial. This procedure was repeated for ten trials. Reaction time in milliseconds was recorded at the point of pressing the space bar and the number of correctly identified visual changes (percentage) was noted on paper sheet.

ADD FIGURE 1 ABOUT HERE

\section{The Corsi blocks task}

The design and procedure was adapted from Vandierendonck et al. (2004). A computerised version of the Corsi blocks task was created using E-Prime 2.0 and involved presentation of 9 blue squares on a computer screen. The blue squares changed to yellow in sequence and participants were required to hold the sequence in mind before replicating it with the computer mouse. The sequence ran to completion before participants were able to begin replicating the pattern. Each square held the yellow colour for one second before reverting to blue and shifting to the next square (with no ISI).

There were two experimental conditions, forward and backward. In the forward condition, participants were required to recall the sequence in the same order, 
by using the mouse to click on the previously highlighted squares. The trial began with a sequence length of two squares with each sequence presented twice. The number of squares increased by one in each subsequent set of two trials. The full set of trials (for both forward and backward conditions) terminated after two trials of eight squares, with the backward condition requiring recall of squares in reverse order. In both conditions, participants were scored one point every time they correctly recalled a square in the sequence. These points were taken up until the trial terminated. This process was recording the accuracy of correctly recalled squares in the sequence and was taken from experimental scripts and data managed by E-Prime 2.0 .

\section{Word and alpha span task}

The design and procedure was the same as the one adopted from Craik (1986). Each task consisted of 14 lists of English concrete nouns. The word lists progressively increased by one extra word in each list, with two words as the start point. The words were read out loud by the experimenter at 1 word per second. In the word span task, the participants' aim was to recall the words in the same order. Once the participant had made errors with both lists of the same list length, the task stopped.

In the alpha span task the same procedure applied with the exception that the participant had to recall word lists in alphabetical order. One point was awarded for each item recalled in the correct position, which generated a final score for each task. Rules were strictly applied such that, once an incorrect or misplaced item was recalled, no further points were awarded for that trial.

Cattell's Culture Fair Test (Scale 2 form A; Institute for Personality and Ability Testing, 1971): This test a standardized measure of non-verbal fluid 
intelligence, included in order to address whether any group differences on the tests may be explained on the basis of differences in general cognitive ability. This IQ test has been widely used and has good concept and concrete validity scores, (.81 and .70 respectively); test-retest, internal and external reliability scores of .73, .76, and .67 respectively.

\section{Design}

This study consisted of a mixed factorial design, with the between-subject factor being language group (bilinguals vs monolinguals). All participants completed the same tasks: The change blindness task, the Corsi blocks task, the word and alpha span task and the Culture Fair Test. The dependent variables were the cognitive outcomes in terms of response time and levels of accuracy on the measures tested for visuo-spatial memory and verbal memory. Non-verbal reasoning scores were used as a co-variate.

\section{Results}

There was no statistical age difference between the two groups, $t(58)=1.85, p=.91$. Bilingual participants scored higher than monolinguals on the background measure of non-verbal reasoning, the Cattell's Culture Fair Test (bilinguals: mean $=28.5 / 46$, $\mathrm{SD}=5.3$; monolinguals: mean $=26.3 / 46, \mathrm{SD}=2.5$ ). An independent-sample $t$-test revealed that the difference in performance was just significant, $t(58)=2.029, p=.05$. Sampling therefore included a non-verbal intelligence bias in favour of the bilingual group. In order to ensure that group differences in later analyses did not directly stem from differences in non-verbal intelligence, the Culture Fair score was therefore included as a covariate in all analyses of visuo-spatial and verbal memory task performance. 


\section{Visuo-spatial memory}

Reaction time (RT) and mean accuracy (percent correct; CR) in the change blindness task and the data scores in the Corsi Block tasks (forward and backwards) are shown in Tables 2 and 3. All means have been adjusted for differences in the non-verbal reasoning task.

ADD TABLE 1 and 2 ABOUT HERE

For the change blindness task (Figures 2 and 3), analysis of covariance revealed a significant effect of Group for RT, $F(1,57)=9.68, p=.003, \eta_{p}^{2}=.15$, and for accuracy, $F(1,57)=13.61, p=.001, \eta_{p}{ }^{2}=.19$. Bilinguals were on average 2.9 seconds faster and $11 \%$ more accurate than monolinguals. We also analysed arcsine transformed accuracy scores to address the possibility that the distribution of these data violated parametric assumptions. Results were entirely consistent with the untransformed data $\left(\mathrm{F}(1,57)=15.36, \mathrm{p}<.001, \eta_{p}^{2}=.21\right)$.

For the Corsi block task forward and backward (Figure 4), bilinguals scored on average 2.3 points higher than monolinguals. Analysis of covariance revealed that this difference in performance was statistically significant for both conditions, forward $F(1,57)=9.53, p=.003, \eta_{p}{ }^{2}=.14$, and backward $F(1,57)=6.659, p=.012, \eta_{p}{ }^{2}=.11$. 


\section{Verbal memory - The word and alpha span task}

The mean accuracy (Percent CR) in word and alpha span tasks are shown in Table 3. Bilinguals and monolinguals had comparable performance when required to recall the words presented in the task, both in the same order, $F(1,57)=.37, p=.543, \eta_{p}^{2}=.007$, and in alphabetical order $F(1,57)=3.23, p=.077, \eta_{p}{ }^{2}=.05$. However, although both results were statistically non-significant, it is worth noting that bilinguals were more accurate than monolingual speakers (bilingual mean accuracy $=32.6$, SD 9.6; monolingual mean accuracy $=28.7, \mathrm{SD} 6.9$ ), the reverse direction of the prediction from the Luo et al. (2013) study that bilinguals should exhibit a disadvantage.

ADD TABLE 3 ABOUT HERE

Taken together, these results suggest that bilinguals have an advantage over monolinguals in terms of visuo-spatial memory but verbal memory performance is comparable in the two groups. In the change blindness task, the results suggest that bilinguals are not only faster than monolinguals but also more accurate. For the Corsi block task, bilinguals' performance was again better than monolinguals' as 
demonstrated by their higher scores in the forward and backward conditions, irrespective of any group differences in non-verbal reasoning.

\section{Correlation analyses of Corsi block and change blindness performance}

In order to investigate the extent to which the bilinguals' advantage in the Corsi block and change blindness tasks was associated with the same cognitive mechanism(s), we carried out bivariate correlation between the tests. With data collapsed across groups $(\mathrm{N}=60)$ significant correlation between change blindness $\mathrm{RT}$ and Corsi block forward $(r=-.34, p=.008)$ and backward $(r=-.29, p=.024)$ were observed. Change blindness accuracy was significantly correlated with Corsi block forward $(\mathrm{r}=.28, \mathrm{p}=.029)$ but not backward $(\mathrm{r}=-.22, \mathrm{p}=.095)$. Within each group $(\mathrm{N}=30)$, the correlations were non-significant ( $\mathrm{p}>.2$ in all cases). Therefore, despite reaching conventional statistical thresholds when computed across all participants, these small moderate sized correlation coefficients indicate very limited proportion of shared variance $(<12 \%)$ in performance across these tests.

\section{Correlation analyses between verbal and non-verbal tasks across the two groups}

Bivariate correlations were carried out to investigate possible links between the visual tasks (Corsi Block and Change Blindeness) and the verbal tasks (Word and Alpha span). For the bilingual group, reaction time in the Change Blindness task correlated significantly with the Word Span $(\mathrm{r}=-.67, \mathrm{p}<.001)$ and the Alpha span $(\mathrm{r}=-$ $.55, \mathrm{p}=.002)$. In monolinguals, the correlation was significant for Word $\operatorname{span}(\mathrm{r}=-45$, $\mathrm{p}=.012)$ but not for Alpha span $(\mathrm{r}=-.24, \mathrm{p}=.20)$. However, employing the Fisher $\mathrm{r}-\mathrm{to}-\mathrm{z}$ transformation, these correlations did not differ significantly from each other (at $\mathrm{p}=.05$, two-tailed). 
In addition, the Word span and Alpha span correlated significantly with Corsi Block forwards performance, but only in the bilingual group $(\mathrm{r}=.37, \mathrm{p}=.044 ; \mathrm{r}=.51$, $\mathrm{p}=.004$, respectively). In monolinguals these correlations were low and non significant ( $p>.5$ in both cases). No significant correlations between these verbal and non verbal tasks were found in either group ( $\mathrm{p}>.1$ in all cases). Direct comparison of the correlation coefficients across groups did not reveal any significant effects at $\mathrm{p}=.05$, two-tailed.

In summary, the correlations between verbal and non-verbal tasks were stronger within the bilingual group, but the difference in the size of the effects were not statistically reliable. 


\section{Discussion}

The present study compared adult proficient bilinguals, who acquired English in their late childhood, with native English monolingual speakers on a range of measures of cognitive performance. The change blindness task and the Corsi blocks task measured visuo-spatial memory. The word and alpha span task measured participants' verbal memory. Finally, the Cattell's Culture Fair Test measured non-verbal reasoning.

The primary rationale for this study was to assess and build on recent research by Luo et al. (2013), who identified a bilingual advantage in visuo-spatial ability measured with the Corsi blocks task. However, in order to address the claim that the bilingual advantage, where observed, may be the result of task artefact (Paap et al., 2014), we extended our exploration by adding another task, the change blindness task (Rensink, 2002). This task was also added for arguably embracing a more reliable ecological validity.

Results revealed that the bilingual adult speakers were significantly faster and more accurate than monolinguals in processing visuo-spatial materials on both tasks (i.e., Corsi blocks and change blindness). These differences held even when between group variability in non-verbal reasoning (measured by Cattell's Culture Fair performance) was partialled out. On the one hand, the results are consistent with the view that the non-random allocation of participants to bilingual and monolingual groups risks sampling confounds. Here, we found a reliable advantage for non-verbal reasoning in the bilingual group. On the other hand, controlling for this difference, we still obtained findings consistent with those of Luo and colleagues, that a bilingual advantage can be observed in processing visuo-spatial information in working memory. 
Although significant correlations between performance on the change blindness and Corsi blocks task were observed with data collapsed across groups, the relationships were low and non-significant at the group level. This observation challenges the claim that the tests assess the same underlying cognitive mechanisms. Consistent with both overlapping and distinct mechanisms serving performance on these and similar tasks, functional neuroimaging data indicates common fronto-parietal involvement but also task specific recruitment. For example, Pessoa and Ungerleider (2004) revealed cerebellar, pulvinar and inferior temporal involvement in addition to predicted fronto-parietal recruitment during change detection. Corsi block task performance is also associated with a frontoparietal network (e.g., Toepper et al, 2010), but recruitment of the pulvinar, inferior temporal gyrus and cerebellum has not been reported.

The change blindness task may place disproportionate demands on the deployment of visual attention (i.e., towards the relevant location within a spatial array) necessary for successful performance. This deployment may be facilitated by inferior temporal visual processing areas, with change detection also benefiting from cerebellar and pulvinar co-involvement (Pessoa \& Ungerleider, 2004). Given that attention is automatically drawn to visually salient stimuli, in comparison to the change blindness task, Corsi blocks performance is more likely to be contingent upon bottom up deployment of visual attention. Thus, while both the change blindness and Corsi blocks tasks recruit mechanisms serving visuo-spatial working memory, the moderate/low correlations reported here are consistent with functionally dissociated regions, with different levels of dependence on these regions required for successful performance across tasks. That a bilingual advantage was observed in both tasks indicates that general spatial working memory mechanisms (i.e., common to both 
tasks) may have been strengthened via processes associated with second language acquisition.

Correlations between the verbal (word and alpha span) and non-verbal (change blindness and Corsi block) tasks were generally stronger in the bilingual group, perhaps indicating a strengthening of domain-general executive mechanisms serving both visual and verbal modalities in bilinguals. Nevertheless, if this were the case, one might intuitively expect stronger correlations between the more demanding tasks (Corsi backwards and alpha span), but this was not observed in the current study. Additionally, given that direct comparison of correlations across groups revealed no significant effects, this possibility remains speculative.

These findings are consistent and comparable to Bialystok's (1992) findings using the embedded figure task where bilinguals identified the complex image within a simple image quicker than monolinguals. In the change blindness task participants are actively looking for a visual change while holding in memory the preceding visual stimuli, then - if successful - selectively attending to the target information prior to producing the correct response (Ma, Xu, Wong, Jiang, \& Hu, 2013). Change blindness is assumed to reflect a failure to store task relevant visual stimuli in shortterm memory (Rensink et al., 1997; Rensink, 2002) and the current research exemplifies that bilinguals are better at this process than monolinguals.

The Corsi blocks task (forwards and backwards) generated consistent results, whereby the bilingual speakers outperformed monolinguals in both forward and backward conditions. These results are comparable to those reported by Luo and colleagues (2013) who also found that bilinguals outperformed monolinguals on both forward and backward conditions. Our results therefore confirm the surprising evidence of a group advantage for bilinguals in visuo-spatial memory. 
Previous research has suggested that while the forwards task draws on spatialsequential resources, the backwards task may place relatively greater demand on executive and distinctively visual processes (Bacon, Parmentier \& Barr, 2013). The advantage may not lie in the bilingual speakers' enhanced ability to inhibit the misleading spatial cue, but in their ability to flexibly manage attention across a complex set of rapidly changing task demands (Bialystok et al., 2004). The findings from this task are also supported by Hilchey and Klein (2011) who suggested that bilinguals react quicker than monolinguals to visual stimuli when faced with congruent (easy) and incongruent (difficult) tasks.

Again, the results from the Corsi blocks task are consistent with the prediction that bilinguals have an advantage in visuo-spatial memory, in comparison to monolingual speakers. This suggests that the bilingual advantage is present in this area of cognitive performance, indicating that a strong executive control component is apparent in visuo-spatial memory (Luo et al., 2013).

When considering the verbal measures, there was no significant difference between language groups in both the word span task (simple condition) and the alpha word span task (difficult condition). It is worth noting that previous research indicates a bilingual disadvantage on simple verbal memory tasks (e.g., Bialystok et al., 2009; Gollan et al., 2005; Luo et al., 2013). In Luo et al.'s study, a large proportion of the participants used English as their dominant and most used language, but in our study all participants learned English as their second language. Thus, our assessment of verbal memory (the word and alpha span task) focused on L2, but Luo et al. assessed L1. Whether this difference in participant characteristics explains the verbal processing disparity across studies cannot be confirmed but warrants further investigation. 
The findings in the current study provide further empirical evidence for cognitive bilingual advantages in processing visuo-spatial information in working memory. Despite scepticism concerning the extent to which the 'bilingual advantage' extends to other cognitive abilities (e.g., Paap et al., 2013), we observed a differential benefit in working memory for spatial materials over verbal materials in bilinguals compared to the monolingual group.

. While recruiting bilinguals and monolinguals from an (otherwise relatively homogeneous) undergraduate population could, as we suspected, involve sampling confounds (in this case, of a non-verbal reasoning ability advantage for bilinguals), these were not sufficient to explain the group differences in visuo-spatial working memory. It is possible that socio-economic status may have contributed, at least in part, to the group differences presented here, and further work may clarify the relevance of this potential confound to the findings reported here and in the wider literature.

Transfer effects associated with multi-language learning prompt the need for a mechanistic account either through which 1) bilingualism produces enhancements to mechanisms that are also involved in visuo-spatial working memory; or 2) bilinguals gain experiences which separately enhance independent mechanisms of language processing and visuo-spatial working memory. Executive functions provide a possible candidate for the common mechanisms in the former view, though we did not find a differential benefit in those memory conditions thought to differentially rely on executive functions. For the latter view, it is as yet unclear what aspects of the experience of bilingualism would cause independent enhancement of visuo-spatial skills. 
Our results suggest the need for a larger scale investigation which should incorporate participants from several age-groups with the aim of building contrasting developmental trajectories for verbal and visuo-spatial abilities across the lifespan. This is particularly relevant, because executive functions show extended development across childhood. To the extent that they are the locus of transfer effects, a developmental framework should give deeper insights into the origin of the bilingual advantage, in those skills where it is present.

In summary, we have provided further evidence that nonverbal tasks requiring attentional control are performed more efficiently by bilingual speakers than monolinguals, perhaps due to increased demands on inhibitory processing associated with managing and switching between two active languages (Bialystok et al., 2009; Green, 1986,1998; although see Duñabeitia et al. (2014) or Paap et al. (2013) for alternative findings and explanations).

In particular, performance on the change blindness task reported in the present study indicates that the ability to produce purposive goal-directed behaviours in complex visual environments may benefit via the developmental process of becoming bilingual and maintaining that proficiency in two or more languages. It is important that the public, in particular parents and educators, are aware of the potential importance of speaking more than one language on the development and maintenance of cognitive abilities. 


\section{REFERENCES}

Bacon, A. M., Parmentier, F. B., \& Barr, P. (2013). Visuospatial memory in dyslexia: Evidence for strategic deficits. Memory, 21(2), 189-209.

Baddeley, A. D. (1986). Working memory. Oxford: Oxford University Press.

Baddeley, A. (1992). Working memory. Science, 255(5044), 556-559.

Bialystok, E. (1982). On the relationship between knowing and using linguistic forms1. Applied Linguistics, 3(3), 181-206.

Bialystok, E. (1992). Symbolic representation of letters and numbers. Cognitive Development, 7(3), 301-316.

Bialystok, E. (2001). Bilingualism in development: Language, literacy, and cognition. Cambridge University Press.

Bialystok, E. (2005). Consequences of bilingualism for cognitive development. Handbook of bilingualism, 417-432.

Bialystok, E. (2007). Acquisition of literacy in bilingual children: A framework for research. Language learning, 57(s1), 45-77.

Bialystok, E. (2009). Bilingualism: The good, the bad, and the indifferent.Bilingualism: Language and Cognition, 12(01), 3-11.

Bialystok, E., \& Feng, X. (2011). Language proficiency and its implications for monolingual and bilingual children. Language and literacy development in bilingual settings, 121-138.

Bialystok, E., \& Martin, M. M. (2004). Attention and inhibition in bilingual children: Evidence from the dimensional change card sort task. Developmental science, 7(3), 325-339. 
Bialystok, E., Craik, F., \& Luk, G. (2008). Cognitive control and lexical access in younger and older bilinguals. Journal of Experimental Psychology: Learning, Memory, and Cognition, 34(4), 859.

Bialystok, E., Craik, F. I., Green, D. W., \& Gollan, T. H. (2009). Bilingual minds. Psychological Science in the Public Interest, 10(3), 89-129.

Bialystok, E., Craik, F. I., Klein, R., \& Viswanathan, M. (2004). Bilingualism, aging, and cognitive control: evidence from the Simon task. Psychology and aging, 19(2), 290.

Bialystok, E., Barac, R., Blaye, A., \& Poulin-Dubois, D. (2010). Word mapping and executive functioning in young monolingual and bilingual children. Journal of Cognition and Development, 11(4), 485-508.

Carlson, S. M., \& Meltzoff, A. N. (2008). Bilingual experience and executive functioning in young children. Developmental science, 11(2), 282-298.

Craik, F. I. (1986). A functional account of age differences in memory. Human memory and cognitive capabilities: Mechanisms and performances, 409-422.

Craik, F. I., Bialystok, E., \& Freedman, M. (2010). Delaying the onset of Alzheimer disease Bilingualism as a form of cognitive reserve. Neurology, 75(19), 17261729.

Crystal, D. (1997, November). Language: Medium, barrier, or Trojan horse. In Conference on cultural diplomacy at the crossroads: Cultural relations in Europe and the wider world. Wilton House (Vol. 26). 
de Bruin, A., Treccani, B., \& Della Sala, S. (2015). Cognitive Advantage in Bilingualism An Example of Publication Bias? Psychological science, 26(1), 99-107.

Duñabeitia, J. A., Hernández, J. A., Antón, E., Macizo, P., Estévez, A., Fuentes, L. J., \& Carreiras, M. (2014). The inhibitory advantage in bilingual children revisited: myth or reality? Experimental psychology, 61(3), 234.

Filippi, R., Richardson, F. M., Dick, F., Leech, R., Green, D. W., Thomas, M. S., \& Price, C. J. (2011). The right posterior paravermis and the control of language interference. The Journal of Neuroscience, 31(29), 10732-10740.

Filippi, R., Leech, R., Thomas, M. S., Green, D. W., \& Dick, F. (2012). A bilingual advantage in controlling language interference during sentence comprehension. Bilingualism: Language and Cognition, 15(04), 858-872.

Filippi, R., Morris, J., Richardson, F. M., Bright, P., Thomas, M. S., Karmiloff-Smith, A., \& Marian, V. (2015). Bilingual children show an advantage in controlling verbal interference during spoken language comprehension. Bilingualism: Language and Cognition, 18(03), 490-501.

Gollan, T. H., \& Acenas, L. A. R. (2004). What is a TOT? Cognate and translation effects on tip-of-the-tongue states in Spanish-English and tagalog-English bilinguals. Journal of Experimental Psychology: Learning, Memory, and Cognition, 30(1), 246.

Gollan, T. H., \& Kroll, J. F. (2001). Bilingual lexical access. The handbook of cognitive neuropsychology: What deficits reveal about the human mind, 321345.

Gollan, T. H., Montoya, R. I., \& Werner, G. A. (2002). Semantic and letter fluency in Spanish-English bilinguals. Neuropsychology, 16(4), 562. 
Gollan, T. H., Montoya, R. I., Fennema-Notestine, C., \& Morris, S. K. (2005).

Bilingualism affects picture naming but not picture classification. Memory \& Cognition, 33(7), 1220-1234.

Green, D. W. (1986). Control, activation, and resource: A framework and a model for the control of speech in bilinguals. Brain and language, 27(2), 210-223.

Green, D. W. (1998). Mental control of the bilingual lexico-semantic system.Bilingualism: Language and cognition, 1(02), 67-81.

Grimes, J. (1996). On the failure to detect changes in scenes across saccades.

Grosjean, F. (2010) Bilingual: Life and Reality, Harvard University Press.

Hakuta, K., \& Diaz, R. M. (1985). The relationship between degree of bilingualism and cognitive ability: A critical discussion and some new longitudinal data. Children's language, 5, 319-344.

Hilchey, M. D., \& Klein, R. M. (2011). Are there bilingual advantages on nonlinguistic interference tasks? Implications for the plasticity of executive control processes. Psychonomic bulletin \& review, 18(4), 625-658.

Institute for Personality and Ability Testing. (1973). Measuring intelligence with the Culture Fair tests. Champaign, Illinois: Institute for Personality and Ability Testing.

Hollingworth, A., \& Henderson, J. M. (2002). Accurate visual memory for previously attended objects in natural scenes. Journal of Experimental Psychology: Human Perception and Performance, 28(1), 113.

Levin, D. T., \& Simons, D. J. (1997). Failure to detect changes to attended objects in motion pictures. Psychonomic Bulletin \& Review, 4(4), 501-506. 
Luo, L., Craik, F. I., Moreno, S., \& Bialystok, E. (2013). Bilingualism interacts with domain in a working memory task: Evidence from aging. Psychology and aging, 28(1), 28.

Luo, L., Luk, G., \& Bialystok, E. (2010). Effect of language proficiency and executive control on verbal fluency performance in bilinguals. Cognition, 114(1), 29-41.

Ma, L. Q., Xu, K., Wong, T. T., Jiang, B. Y., \& Hu, S. M. (2013).

Change blindness images. Visualization and Computer Graphics, IEEE Transactions on, 19(11), 1808-1819.

Martin-Rhee, M. M., \& Bialystok, E. (2008). The development of two types of inhibitory control in monolingual and bilingual children. Bilingualism: Language and Cognition, 11(01), 81-93.

Morton, J. B., \& Harper, S. N. (2007). What did Simon say? Revisiting the bilingual advantage. Developmental Science, 10(6), 719-726.

Milner, B. (1971). Interhemispheric differences in the localization of psychological processes in man. British Medical Bulletin.

Mitroff, S. R., Simons, D. J., \& Levin, D. T. (2004). Nothing compares 2 views: Change blindness can occur despite preserved access to the changed information. Perception \& Psychophysics, 66(8), 1268-1281.

Miyake, A., Friedman, N. P., Emerson, M. J., Witzki, A. H., Howerter, A., \& Wager, T. D. (2000). The unity and diversity of executive functions and their contributions to complex "frontal lobe" tasks: A latent variable analysis. Cognitive psychology, 41(1), 49-100. 
Norman, D., \& Shallice, T. (1980). Attention to Action: Willed and Automatic Control of Behaviour, CHIP Report 99. San Diego: University of California.

Norman, D. A., \& Shallice, T. (1986). Attention to action (pp. 1-18). Springer US.

Paap, K. R., \& Greenberg, Z. I. (2013). There is no coherent evidence for a bilingual advantage in executive processing. Cognitive Psychology, 66(2), 232-258.

Paap, K. R., Johnson, H. A., \& Sawi, O. (2014). Are bilingual advantages dependent upon specific tasks or specific bilingual experiences?. Journal of Cognitive Psychology, 26(6), 615-639.

Peal, E., \& Lambert, W. E. (1962). The relation of bilingualism to intelligence. Psychological Monographs: general and applied, 76(27), 1-23.

Pessoa, L., \& Ungerleider, L. G. (2004). Neural correlates of change detection and change blindness in a working memory task. Cerebral Cortex, 14(5), 511-520.

Rensink, R. A. (2002). Change detection. Annual review of psychology, 53(1), 245 277.

Rensink, R. A., O'Regan, J. K., \& Clark, J. J. (1997). To see or not to see: The need for attention to perceive changes in scenes. Psychological science, 8(5), 368373.

Saer, D. J. (1923). The effect of bilingualism on intelligence. British Journal of Psychology. General Section, 14(1), 25-38.

Schneider, W., Eschman, A., \& Zuccolotto, A. (2002) E-Prime User's Guide. Pittsburgh: Psychology Software Tools Inc.

Simons, D. J., \& Levin, D. T. (2003). What makes change blindness interesting?. Psychology of Learning and Motivation, 42, 295-322. 
Simons, D. J., \& Rensink, R. A. (2005). Change blindness: Past, present, and future. Trends in cognitive sciences, 9(1), 16-20.

Toepper, M., Gebhardt, H., Beblo, T., Thomas, C., Driessen, M., Bischoff, M., ... \& Sammer, G. (2010). Functional correlates of distractor suppression during spatial working memory encoding. Neuroscience, 165(4), 1244-1253.

Vandierendonck, A., Kemps, E., Fastame, M. C., \& Szmalec, A. (2004). Working memory components of the Corsi blocks task. British Journal of Psychology, 95(1), 57-79.

Vega-Mendoza, M., West, H., Sorace, A., \& Bak, T. H. (2015). The impact of late, non-balanced bilingualism on cognitive performance. Cognition, 137, 40-46.

\section{TABLES}

Table 1. Reaction times and percent correct responses in the change blindness task. Standard deviations in brackets. All means have been adjusted for differences in the non-verbal reasoning task. 
Table 2. Ability scores in the Corsi-Blocks forward and backward tasks. Standard deviations in brackets. All means have been adjusted for differences in the non-verbal reasoning task.

\begin{tabular}{lcc} 
& Bilinguals & Monolinguals \\
& Score (SD) & Score (SD) \\
\hline \hline & & \\
Corsi-Blocks Forward & $20.9(3.2)$ & $18.4(3.0)$ \\
Corsi-Blocks Backward & $19.8(3.0)$ & $17.8(3.0)$ \\
& & \\
\hline \hline
\end{tabular}

\begin{tabular}{lllll}
\hline \hline & \multicolumn{2}{c}{ Bilinguals } & \multicolumn{2}{c}{ Monolinguals } \\
& RT (SD) & CR (SD) & RT (SD) & CR (SD) \\
\hline Change-Blindness Task & $12.6(3.9)$ & $92 \%(9.1)$ & $15.6(3.2)$ & $81 \%(12.5)$ \\
\hline \hline
\end{tabular}

\begin{tabular}{lllll}
\hline \hline & \multicolumn{2}{c}{ Bilinguals } & \multicolumn{2}{c}{ Monolinguals } \\
& RT (SD) & CR (SD) & RT (SD) & CR (SD) \\
\hline Change-Blindness Task & $12.6(3.9)$ & $92 \%(9.1)$ & $15.6(3.2)$ & $81 \%(12.5)$ \\
\hline \hline
\end{tabular}


Table 3. Ability scores in the word and alpha span tasks. Standard deviations in brackets. All means have been adjusted for differences in the non-verbal reasoning task.

\begin{tabular}{lcc}
\hline \hline & Bilinguals & Monolinguals \\
& Score $(S D)$ & Score $(S D)$ \\
\hline Word Span & $33.8(10.0)$ & $32.1(9.9)$ \\
Alpha Span & $32.6(9.6)$ & $28.7(6.9)$ \\
& & \\
\hline
\end{tabular}



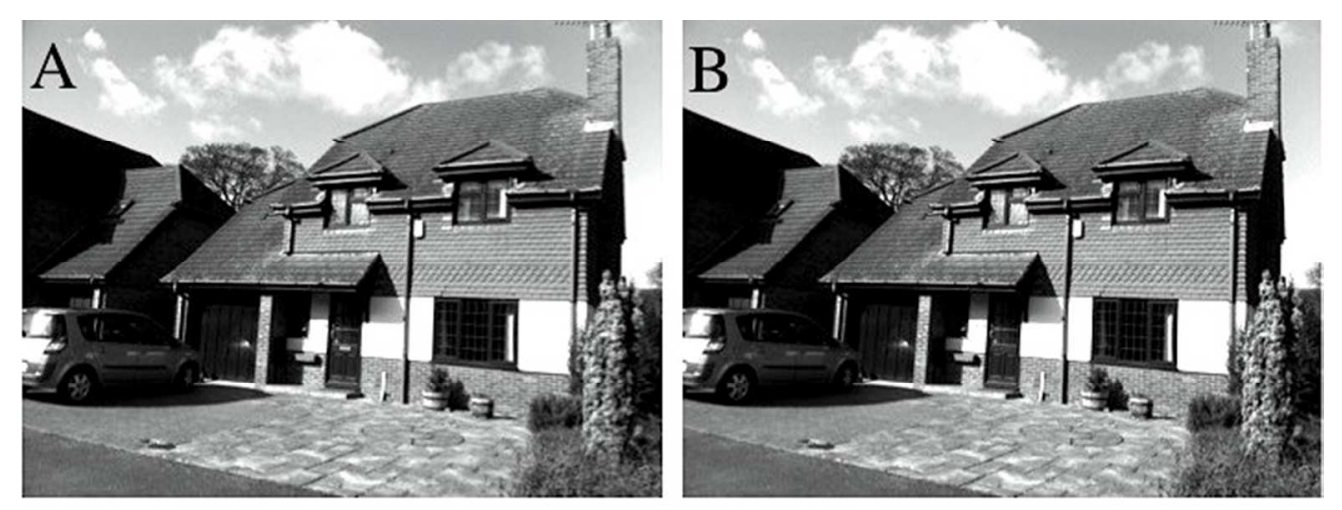

$249 \times 92 \mathrm{~mm}(72 \times 72 \mathrm{DPI})$ 
Change Blindness Task (RT)

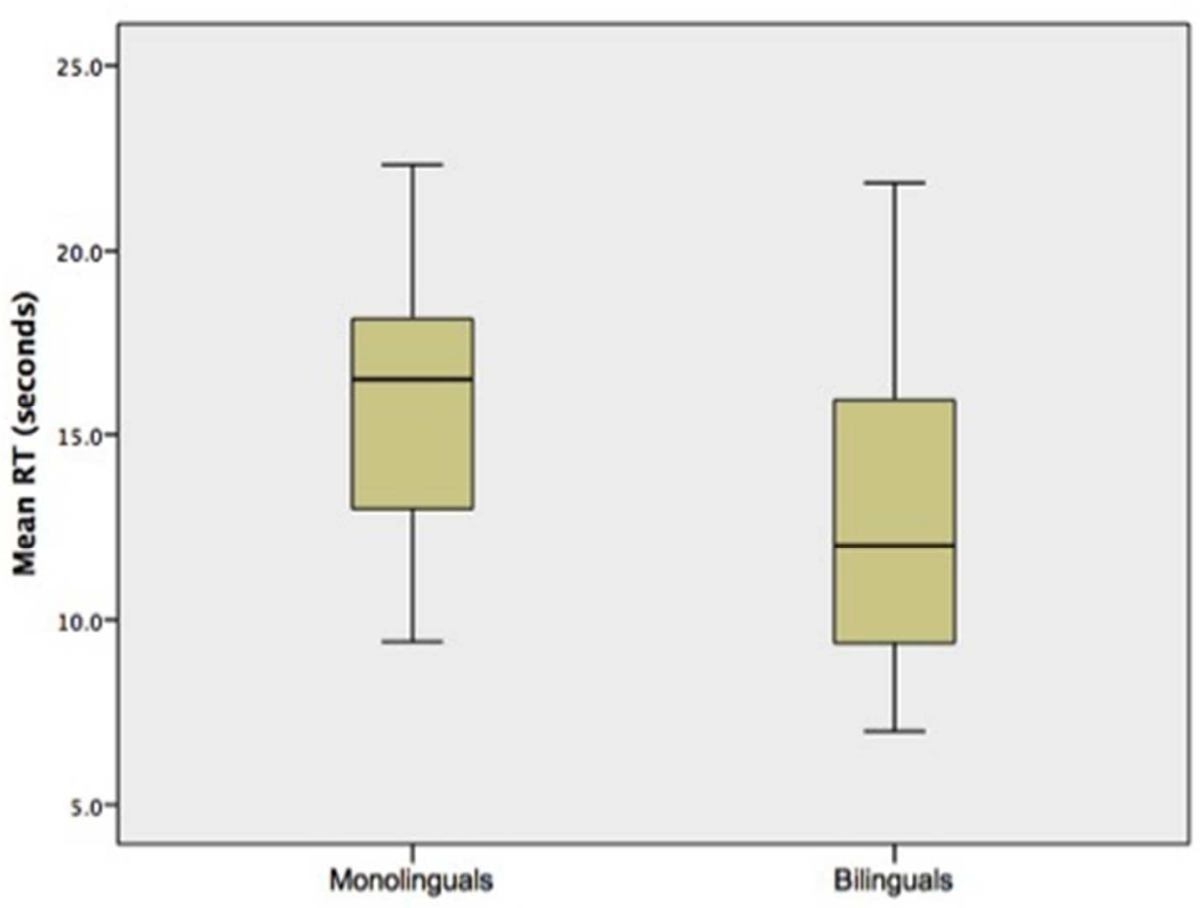

$165 \times 153 \mathrm{~mm}(72 \times 72 \mathrm{DPI})$ 


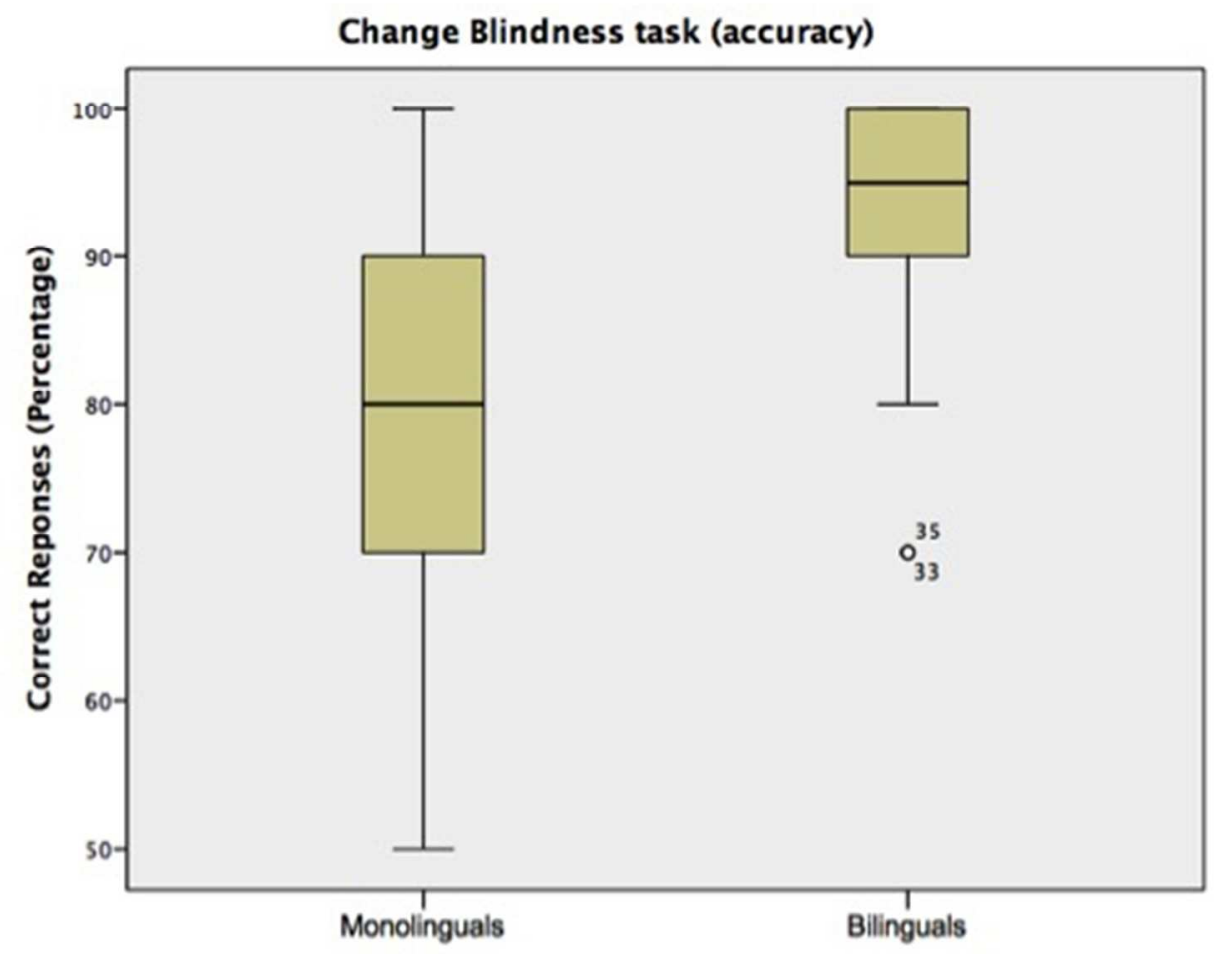

$165 \times 135 \mathrm{~mm}(72 \times 72 \mathrm{DPI})$ 


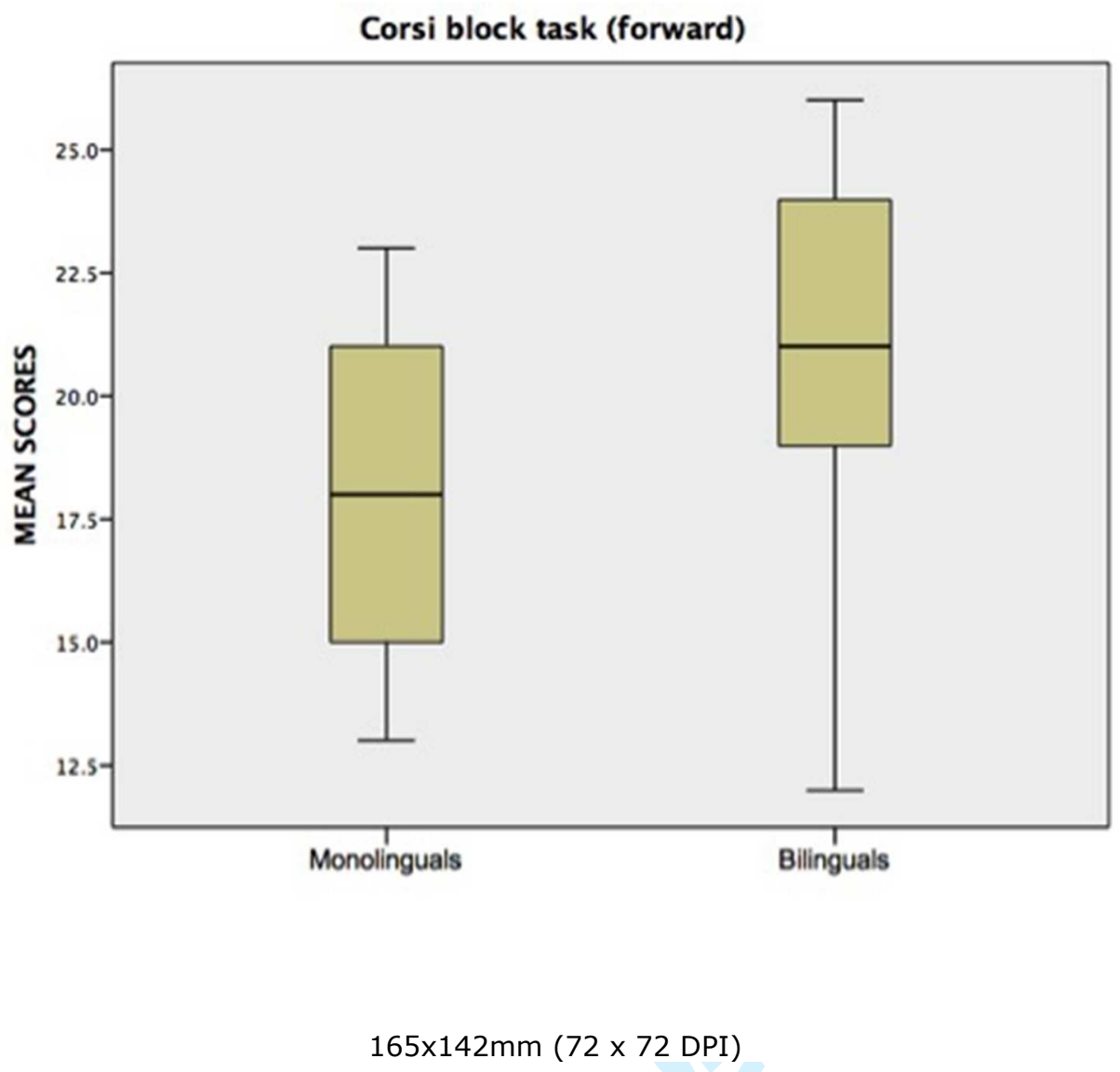

1

2

3

4

5

6

10

11

12

13

14

15

16

17

18

19

20

21

22

23

24

25

26

27

28

29

30

31

32

33

34

35

36

37

38

39

40

41

42

43

44

45

46

47

48

49

50

51

52

53

54

55

56

57

58

59

60

Cambridge University Press 


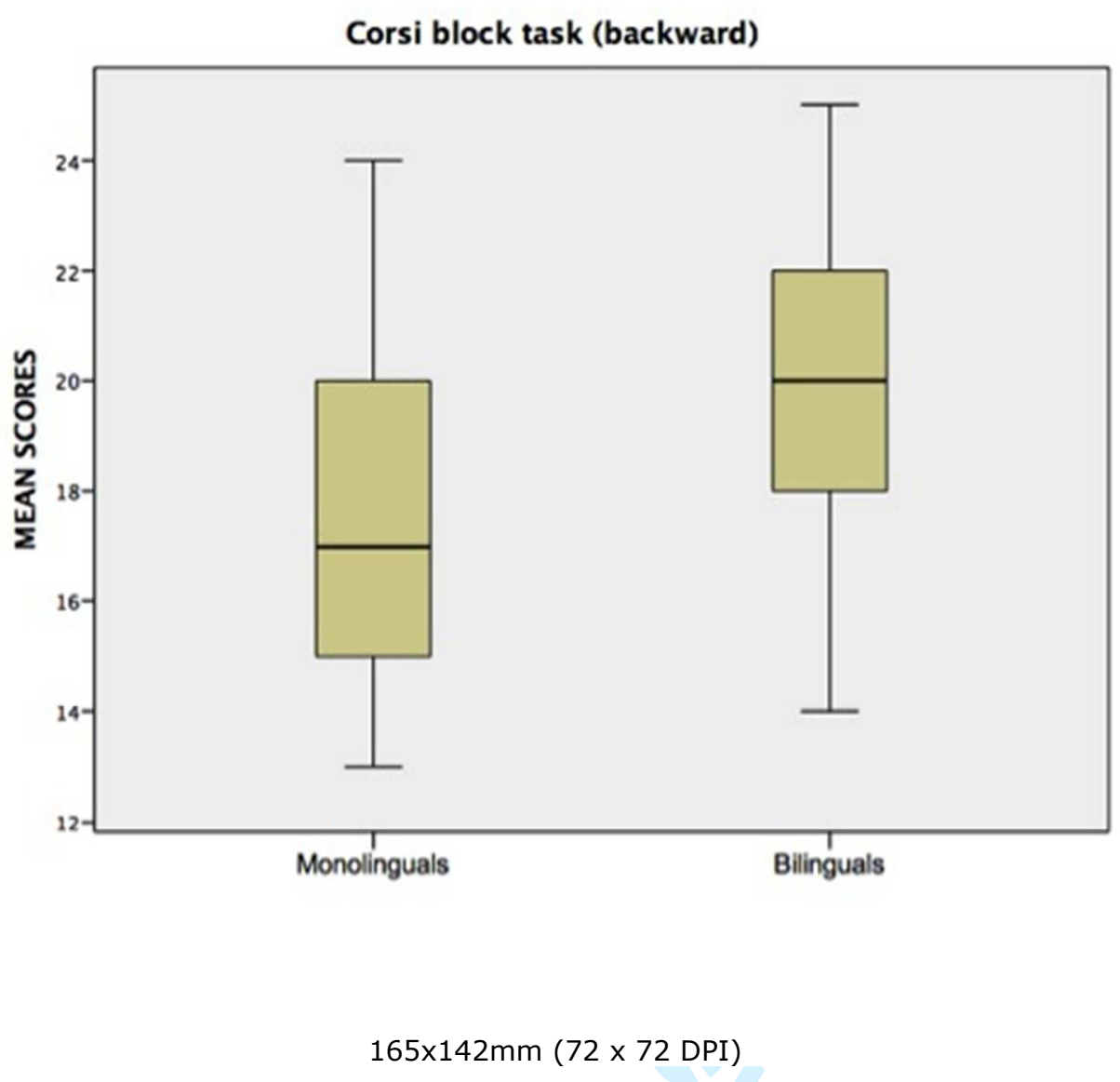

1

2

3

4

5

6

7

8

9

10

11

12

14

15

16

17

18

19

20

21

22

23

24

25

26

27

28

29

30

31

32

33

34

35

36

37

38

39

40

41

42

43

44

45

46

47

48

49

50

51

52

53

54

55

56

57

58

59

60

Cambridge University Press 